\title{
The least uncomfortable journey from A to B
}

\author{
D. Anderson ${ }^{\mathrm{a})}$ \\ Department of Earth and Space Sciences, Chalmers University of Technology, SE-41296 Göteborg, Sweden \\ M. Desaix \\ Faculty of Textiles, Engineering and Business, University College of Borås, SE-501 90 Borås, Sweden \\ R. Nyquist \\ Department of Earth and Space Sciences, Chalmers University of Technology, SE-412 96 Göteborg, Sweden
}

(Received 19 March 2015; accepted 9 June 2016)

\begin{abstract}
A short introduction is given about direct variational methods and their relation to Galerkin and moment methods, all flexible and powerful approaches for finding approximate solutions to difficult physical equations. An application of these methods is given in the form of the variational problem of minimizing the discomfort experienced during different journeys, between two fixed horizontal points while keeping the travel time constant. The analysis is shown to provide simple, yet accurate, approximate solutions of the problem and illustrates the usefulness and the power of direct variational and moment methods. It also demonstrates the problem of a priori assessing the accuracy of the approximate solutions and illustrates that the variational solution does not necessarily provide a more accurate solution than that obtained by moment methods. (C) 2016 American Association of Physics Teachers.

[http://dx.doi.org/10.1119/1.4955151]
\end{abstract}

\section{INTRODUCTION}

Variational calculus is a classical subject in mathematics with many applications in physics and engineering. ${ }^{1-6}$ Although examples of variational problems were formulated very early in the scientific history (a famous example being Fermat's principle of least time in optics), it is fair to say that variational calculus appeared as a particular mathematical field approximately 300 years ago, when it was developed in order to solve the classical brachistochrone problem by Bernoulli in 1696. It was then found that the optimal function of a variational problem must satisfy a certain differential equation-the Euler-Lagrange variational equation-directly determined by the integrand, the Lagrangian density, of the functional to be optimized. In many cases, however, the Euler-Lagrange equation constitutes a complicated differential equation that does not allow an explicit analytical solution. In such situations, direct variational methods, e.g., the Rayleigh-Ritz method based on trial functions, have been found very useful for finding approximate solutions of the problems. A fact seldom emphasized is that the Rayleigh-Ritz method is closely related to another technique for finding approximate solutions of differential equations, the Galerkin method, which is a particular example of a moment method or the method of weighted residuals.

Although some applications of variational calculus, for example Hamilton's principle, are taught at the undergraduate level, direct variational calculus and moment methods are usually considered subjects more suitable for the graduate level. This is rather unfortunate because the direct variational methods developed from variational calculus (such as the Rayleigh-Ritz optimization), as well as moment methods, are widely used and provide powerful means of obtaining approximate analytical solutions. Many such problems can also be presented at the undergraduate level and can be used to encourage the curiosity and the creativity of the students. The purpose of the present work is to demonstrate the formulation of a variational problem and the application of the Rayleigh-Ritz and moment methods to illustrate the power and flexibility of these methods.

To this end, the problem to be considered is the minimization of the discomfort experienced during a journey along a straight horizontal line from one point to another. Even if the travel time is kept fixed, the distance may still be covered in many different ways. Strong acceleration and deceleration are clearly uncomfortable and as a model for the total discomfort experienced during the journey, a discomfort functional may be defined by integrating the acceleration/ deceleration squared (to avoid cancelation effects) over the total journey. Minimizing this functional while keeping the travel time constant leads to a variational problem that can be solved analytically to give the optimal (i.e., least uncomfortable) velocity as a function of traveled distance. On the other hand, it can be-and has been-argued that changes in acceleration/deceleration, more specifically the time rate of change of the acceleration (the so-called jerk ${ }^{7}$ ), gives rise to even more discomfort than acceleration and deceleration, and it is interesting to reexamine the above problem by defining the discomfort functional in terms of the jerk (squared) instead of the acceleration. The jerk plays an important role in a number of diverse technical applications, such as in the design of a smooth gradual transition of the curvature from a straight path to a circular one in railways and highways, as well as to the generation of flow noise in acoustics. $^{7}$

Although the variational problem corresponding to the acceleration-induced discomfort problem can be solved analytically, it is also interesting to compare with the approximate results obtained using the Rayleigh-Ritz and moment methods. The approximate solutions are found to be in good agreement with the exact solution. On the other hand, the concomitant variational equation for the jerk-induced discomfort is more complicated and it does not seem possible to find an analytical solution. Again, the Rayleigh-Ritz optimization procedure and the moment method are used to find simple approximate solutions. Based on trial functions similar to those used in the acceleration-discomfort problem, it is found that the moment method gives a significantly better result than that of the direct variational approach when compared with the numerically obtained solution. The reason for this somewhat surprising result is explained by the fact that the trial function in the Rayleigh-Ritz approach gives rise to 
a discomfort integral that is close to a divergent limit, whereas the moment approach avoids this problem by using appropriate choices of weight functions.

\section{THE DISCOMFORT FUNCTIONALS}

Consider a journey along a straight and horizontal road from point $A$ to point $B$ with neither speed limits nor fellow travelers, in an ideal car being able to accelerate and decelerate without bounds and to attain any velocities. The journey starts from rest at $\mathrm{A}$ and ends with the car standing still at $\mathrm{B}$. The time $T$ elapsed in going from A to B is given by

$$
T=\int_{0}^{T} d t=\int_{-D}^{+D} \frac{d x}{v(x)},
$$

where $v(x)$ denotes the velocity as a function of distance $x$. Without loss of generality, we have introduced a coordinate system such that point $\mathrm{A}$ corresponds to $x=-D$ and point $\mathrm{B}$ corresponds to $x=+D$, and consequently $v( \pm D)=0$. It is evident that the variation of velocity with time (or distance) may by chosen in many different ways while still giving rise to the same travel time. However, the discomfort experienced will depend on the chosen velocity variation. An unpleasant feature of a journey is strong acceleration/deceleration and a natural measure of the total discomfort during the journey is the discomfort functional

$$
J[v(x)]=\int_{0}^{T} a^{2} d t=\int_{-D}^{+D} v\left(\frac{d v}{d x}\right)^{2} d x,
$$

where $a(t)$ denotes the acceleration, which can be expressed in terms of the velocity as

$$
a(t)=\frac{d v}{d t}=v \frac{d v}{d x}=\frac{1}{2} \frac{d\left(v^{2}\right)}{d x} .
$$

The problem of minimizing $J[v(x)]$ while keeping the travel time constant leads to a variational problem that involves a subsidiary condition (the constant travel time). It can be formulated as

$$
\delta J[v(x)]=\delta \int_{-D}^{+D} v\left(\frac{d v}{d x}\right)^{2} d x=0,
$$

subject to the subsidiary condition

$$
\int_{-D}^{+D} \frac{d x}{v(x)}=T
$$

or, equivalently,

$$
\delta \int_{-D}^{+D} L(v, d v / d x) d x=0,
$$

where the Lagrangian $L(v, d v / d x)$ is given by

$$
L(v, d v / d x)=v\left(\frac{d v}{d x}\right)^{2}+\frac{\lambda}{v}
$$

Here, $\lambda$ plays the role of a Lagrange multiplier that can be determined from the subsidiary condition. A similar, mathematically equivalent problem was analyzed in Ref. 8 . However, the present analysis will pursue the new problem formulation further than in Ref. 8, to obtain a simple exact solution for the variation of distance with time, and also to demonstrate how accurate approximate solutions of the problem can be found using moment methods.

The Euler-Lagrange equation corresponding to the variational problem reads

$$
\frac{\partial L}{\partial v}-\frac{d}{d x} \frac{\partial L}{\partial(d v / d x)}=0
$$

which implies the differential equation

$$
2 v \frac{d^{2} v}{d x^{2}}+\left(\frac{d v}{d x}\right)^{2}+\frac{\lambda}{v^{2}}=0
$$

where $v(0)=v_{m}$ (the unknown maximum value of the velocity), $v( \pm D)=0$, and $d v(0) / d x=0$ (using the fact that the velocity $v(x)$ is symmetric around $x=0)$. The solution of Eq. (9) can be found in implicit form, $x=x(v)$, and is given by ${ }^{8}$

$$
\sqrt{\Lambda}(x / D)=\frac{2}{3}\left(2+v / v_{m}\right) \sqrt{1-v / v_{m}}, \quad 0 \leq x / D \leq 1,
$$

where $\Lambda \equiv \lambda D^{2} / v_{m}^{4}$. It is enough to consider only the interval $0 \leq x / D \leq 1$, with the function $v(x)$ being even. The condition $v(D)=0$ directly determines the normalized Lagrange multiplier to be $\Lambda=16 / 9$. Finally, the integral in Eq. (1) (the subsidiary condition) can be evaluated using the change of variables determined by Eq. (10). The value of the maximum speed $v_{m}$, in terms of travel distance $2 D$ and time $T$, is found to be $v_{m}=3 D / T$. It is interesting to note that Eq. (10) can be inverted to yield

$$
v / v_{m}=2 \cos \left[\frac{2}{3} \arcsin (x / D)\right]-1,
$$

which can be integrated further (note that $v=d x / d t$ ) to give

$$
t / T=\frac{1}{2}+\sin \left[\frac{1}{3} \arcsin (x / D)\right] .
$$

Finally, this expression can be inverted to give the distance $x$ as an explicit third-order polynomial in time $t$

$$
x / D=-1+6(t / T)^{2}-4(t / T)^{3} .
$$

Turning now to the jerk-induced discomfort, it is clear that the corresponding discomfort functional can be defined analogously as

$$
J[v(x)]=\int_{0}^{T} j^{2} d t=\int_{-D}^{+D} \frac{v}{4}\left[\frac{d^{2}\left(v^{2}\right)}{d x^{2}}\right]^{2} d x
$$

where the jerk $j$ has been expressed as

$$
j=\frac{d a}{d t}=\frac{d}{d t}\left[\frac{1}{2} \frac{d\left(v^{2}\right)}{d x}\right]=\frac{v}{2} \frac{d^{2}\left(v^{2}\right)}{d x^{2}} .
$$

The corresponding variational problem thus becomes 


$$
\delta \int_{-D}^{+D}\left\{\frac{v}{4}\left[\frac{d^{2}\left(v^{2}\right)}{d x^{2}}\right]^{2}+\frac{\lambda}{v}\right\} d x=0 .
$$

It is convenient to rewrite Eq. (16) using the variable $V=v^{2}$, in terms of which the variational problem can be written

$$
\delta \int_{-D}^{+D}\left[\frac{\sqrt{V}}{4}\left(\frac{d^{2} V}{d x^{2}}\right)^{2}+\frac{\lambda}{\sqrt{V}}\right] d x=0 .
$$

For Lagrangians of the form $L=L\left(y, d^{2} y / d x^{2}\right)$, the corresponding variational derivative is

$$
\frac{\delta L}{\delta y}=\frac{\partial L}{\partial y}+\frac{d^{2}}{d x^{2}} \frac{\partial L}{\partial\left(d^{2} y / d x^{2}\right)},
$$

which in the present case results in the following variational equation for $v(x)$ :

$$
\frac{d^{2}}{d x^{2}}\left(\sqrt{V} \frac{d^{2} V}{d x^{2}}\right)+\frac{1}{4 \sqrt{V}}\left(\frac{d^{2} V}{d x^{2}}\right)^{2}-\frac{\lambda}{V \sqrt{V}}=0
$$

This equation is equivalent to the Euler-Lagrange equation obtained from Eq. (16) for $v(x)$, but is algebraically simpler. Necessary boundary conditions for Eq. (19) on the interval $0 \leq x \leq D \quad$ are $\quad V(D)=0, \quad V(0)=v_{m}^{2}, \quad$ and $\quad d V(0) / d x$ $=d^{3} V(0) / d x^{3}=0$. The last two boundary conditions, requiring vanishing odd derivatives at $x=0$, are due to the fact that we are looking for an even function on the interval, $-D \leq x \leq+D$. In addition, the journey must start and end with the car at rest, which means that the initial acceleration/ deceleration must be zero in order to have finite jerk at the beginning and end of the journey. This observation [cf. Eq. (3)] provides the additional boundary condition of vanishing acceleration at $x=D$, i.e., $d V(D) / d x=0$. Together, these constitute the five boundary conditions necessary to solve the problem given by the fourth-order differential equation in Eq. (19), including the unknown multiplier $\lambda$. However, Eq. (19) seems too difficult to permit an exact analytical solution, and resort must be taken to approximate analytical and/or numerical methods. Direct variational and moment methods are very powerful, and are often used in situations like this. These approaches and the relation between them will be elaborated in some detail in Sec. III.

\section{RELATION BETWEEN THE RAYLEIGH-RITZ OPTIMIZATION AND THE MOMENT METHODS}

In many variational problems, it turns out that the corresponding Euler-Lagrange equations cannot be solved analytically. In such situations, direct variational methods (such as the Rayleigh-Ritz method) have been found very useful for obtaining approximate solutions of the problems. The basic idea in the Rayleigh-Ritz procedure is simple, but general. Instead of allowing an arbitrary variation $\delta y(x)$ in the functional analysis, the optimum solution is sought among a restricted sub-set of the allowable function space via trial functions of given functional form, but with flexibility incorporated by dependence on a number of parameters $\alpha_{k}$, i.e., $y(x) \rightarrow y_{T}\left(x ; \alpha_{1}, \alpha_{2}, \ldots, \alpha_{n}\right)$. However, trial functions involving free parameters imply that the functional becomes an ordinary function of the parameters $\alpha_{k}$ and the optimization of the functional corresponds to the conditions

$$
\frac{\partial\left\langle L\left(y_{T}\right)\right\rangle}{\partial \alpha_{k}}=0, \quad k=1,2, \ldots, n,
$$

where

$$
\left\langle L\left(y_{T}\right)\right\rangle=\int_{a}^{b} L\left(y_{T}\right) d x
$$

Another way of writing the first variation of the functional is suggestive, given by

$$
\begin{aligned}
\delta \int_{a}^{b} L\left(y_{T}\right) d x= & \int_{a}^{b} \frac{\delta L\left(y_{T}\right)}{\delta y_{T}} \delta y_{T} d x \\
= & \sum_{k=1}^{n}\left(\int_{a}^{b} \frac{\delta L\left(y_{T}\right)}{\delta y_{T}} \frac{\partial y_{T}\left(x ; \alpha_{1}, \alpha_{2}, \ldots, \alpha_{n}\right)}{\partial \alpha_{k}} d x\right) \\
& \times \delta \alpha_{k}=0 .
\end{aligned}
$$

Since the variations $\delta \alpha_{k}$ of the parameters $\alpha_{k}$ are independent, this implies that the restricted optimization condition becomes

$$
\begin{aligned}
& \int_{a}^{b} \frac{\delta L\left(y_{T}\right)}{\delta y_{T}} \frac{\partial y_{T}\left(x ; \alpha_{1}, \alpha_{2}, \ldots, \alpha_{n}\right)}{\partial \alpha_{k}} d x=0 \\
& \quad k=1,2, \ldots, n
\end{aligned}
$$

which is equivalent to Eq. (20), but has the advantage that it can be written as

$$
\int_{a}^{b} R\left[y_{T}\right] w_{k}(x) d x=0, \quad k=1,2, \ldots, n
$$

where $R\left[y_{T}\right]=\delta L\left(y_{T}\right) / \delta y_{T}$ is the residual error function obtained when inserting the trial function in the Euler-Lagrange equation $\delta L(y) / \delta y=0$, and

$$
w_{k}(x)=\frac{\partial y_{T}\left(x ; \alpha_{1}, \alpha_{2}, \ldots, \alpha_{n}\right)}{\partial \alpha_{k}}, \quad k=1,2, \ldots, n .
$$

Note that $y_{T}$ does not satisfy the equation $R\left[y_{T}\right]=0$ since $y_{T}$ is not a solution of $R[y]=0$. In this form, the optimization condition implies the vanishing of certain weighted moments of the Euler-Lagrange equation. This idea is closely related to another, but even more general, approximation method known as the Galerkin method. This method is a special case of the more general moment method or the method of weighted residuals. 5,6 The first part of the Galerkin and moment methods is equivalent to the Rayleigh-Ritz optimization procedure: an approximate solution of a given differential equation is sought in the form of a trial function, of specified $x$-dependence, but with flexibility allowed by including a number of parameters, i.e., $y(x)=y_{T}\left(x ; \alpha_{1}, \alpha_{2}, \ldots, \alpha_{n}\right)$, where $y_{T}\left(x ; \alpha_{1}, \alpha_{2}, \ldots, \alpha_{n}\right)$ satisfies the boundary conditions for all parameters $\alpha_{k}, k=1,2, \ldots, n$. Clearly, this ansatz function in general does not satisfy the considered differential equation $R[y]=0$, and rather gives rise to a residual $R\left[y_{T}(x)\right] \neq 0$. This residual can be made to vanish, however, in a weighted averaged sense, by multiplying it with certain weight functions $w_{k}(x)$ and integrating over the interval

$$
\int_{a}^{b} R\left[y_{T}\left(x ; \alpha_{1}, \alpha_{2}, \ldots, \alpha_{n}\right)\right] w_{k}(x) d x=0, \quad k=1,2, \ldots, n .
$$


This equation provides $n$ relations for determining the $n$ unknown parameters $\alpha_{k}$. In fact, if the original equation originates from a variational problem and the weight functions are taken as $w_{k}=\partial y_{T} / \partial \alpha_{k}$, the corresponding variational and moment equations coincide. ${ }^{5,6}$ However, the moment approach is more flexible than the variational approach, being applicable to a broader range of problems, such as those where a variational reformulation of the original equation is not possible. It also offers some freedom by allowing an unrestricted choice of weight functions. However, in the Galerkin method, the weight function is chosen to be equal to the trial function. ${ }^{5,6}$

\section{SOLUTIONS OF THE DISCOMFORT PROBLEMS}

It is instructive to begin by applying the approximation procedures to the first problem with the acceleration/decelerationbased discomfort functional for which an explicit analytical solution exists for easy comparison. A crucial step in the application of the Rayleigh-Ritz optimization procedure, as well as the moment methods, is the choice of trial function. It requires an intuitive idea of what the functional dependence of the solution should look like and should include some flexibility allowed by free parameters. In the present application, the optimal curve $v(x)$, being zero at $x= \pm D$, should be symmetric around $x=0$ (meaning $d v(0) / d x=0$ ) and should be smooth. A simple possible choice would be a function of the form $v_{T}(x)=v_{m}\left(1-x^{2} / D^{2}\right)^{\alpha}$, containing the two free parameters $v_{m}$ (which determines the maximum velocity during the journey) and $\alpha$ (which determines the steepness of the curve). However, variations with respect to $\alpha$ would lead to a difficult transcendental equation and it is simpler to make a reasonable choice for the value of $\alpha$, leaving only $v_{m}$ to be varied. When inserting a trial function of the suggested form into the discomfort integral, one finds that it only converges if $\alpha+(2 \alpha-2)>-1$ or if $\alpha>1 / 3$. Furthermore, the second part of the variational functional, which determines the travel time, converges only if $\alpha<1$. Thus, it is inferred that $1 / 3<\alpha<1$. A reasonable compromise is then $\alpha=1 / 2$, which also holds the promise of giving rise to simple calculations.

Inserting the trial function $v_{T}(x)=v_{m}\left(1-x^{2} / D^{2}\right)^{1 / 2}$ into the Lagrangian given in Eq. (7) and integrating over the interval $[-D, D]$, one finds

$$
\left\langle L\left(v_{T}\right)\right\rangle=\frac{\pi D}{2}\left(\frac{v_{m}^{3}}{D^{2}}+\frac{2 \lambda}{v_{m}}\right) .
$$

Since $\langle L\rangle$ is now a function of $v_{m}$ alone, optimization with respect to $v_{m}$ yields

$$
\frac{\partial\left\langle L\left(v_{T}\right)\right\rangle}{\partial v_{m}}=0 \Rightarrow \frac{\lambda D^{2}}{v_{m}^{4}} \equiv \Lambda=\frac{3}{2}=1.5,
$$

to be compared with the exact value $\Lambda=16 / 9 \approx 1.78$, for an absolute relative error of $16 \%$. Finally, the problem is closed by solving for $v_{m}$ from the subsidiary condition for the travel time. This gives $v_{m}=\pi D / T$, in good agreement with the exact solution $v_{m}=3 D / T$, the corresponding absolute relative error being only $5 \%$. It is interesting to note that the exact solution gives rise to a smooth journey in the sense that it also possesses finite acceleration at the start and endpoints. In fact, it can be shown that $a(D)=-12 D / T^{2}$. Of the different trial functions of the form $v(x)=v_{m}\left(1-x^{2} / D^{2}\right)^{\alpha}$, only the one corresponding to $\alpha=1 / 2$ has finite and nonzero acceleration at $x=D$. The corresponding acceleration is $a(D)=-\pi^{2} D / T^{2}$, the absolute relative error being $18 \%$.

The same result can also be obtained with the moment equation in the form of Eq. (24). To this end, we use the variational derivative $\delta L / \delta v$ in Eq. (9) as the residual $R[v]$ and choose the weight function according to $w(x)=\partial v_{T} / \partial v_{m}$ $=\left(1-x^{2} / D^{2}\right)^{1 / 2}$, as specified by both the Galerkin method and the Rayleigh-Ritz optimization procedure. This yields

$$
\begin{aligned}
\int_{-D}^{+D} R\left[v_{T}\right] w(x) d x= & \int_{-D}^{+D}\left(2 v_{T} \frac{d^{2} v_{T}}{d x^{2}}+\left(\frac{d v_{T}}{d x}\right)^{2}+\frac{\lambda}{v_{T}^{2}}\right) \\
& \times \frac{\partial v_{T}}{\partial v_{m}} d x=\frac{\pi D}{2}\left(\frac{2 \lambda}{v_{m}^{2}}-\frac{3 v_{m}^{2}}{D^{2}}\right)=0
\end{aligned}
$$

which implies $\Lambda=3 / 2$ as before. However, although the Galerkin method uses the trial function as weight function, the general moment method approach does not restrict the choice of weight function. By inspecting Eq. (9), it is inferred that a suitable choice of weight function would be $w=1-x^{2} / D^{2}$, which has the advantage that it leads to very simple calculations. Indeed, a trivial calculation yields $\Lambda=5 / 3=15 / 9 \approx 1.67$, in good agreement with the exact result for the Lagrange multiplier, the absolute relative error being only $6 \%$. Since the trial function is the same, the maximum speed $v_{m}$ remains the same. This illustrates the important point that the accuracy of a direct variational approximation is very difficult to predict a priori, ${ }^{9}$ and also that the result of a moment method approximation might well (for certain weight functions) be better than that of a variational one, even though the same trial function is used. Finally, we note that the differential equation implied by the trial function $v=d x / d t=v_{m} \sqrt{1-x^{2} / D^{2}}$ can be solved to give the distance $x(t)$ in the explicit form

$$
x / D=\sin \left[\pi\left(\frac{t}{T}-\frac{1}{2}\right)\right] .
$$

A comparison between the exact and approximate solutions is given in Fig. 1 .

Turning finally to the jerk-induced discomfort functional, only an approximate approach seems possible, the variational equation being a complicated fourth-order differential equation. Again, a trial function of the form $v(x)=v_{m}(1$ $\left.-x^{2} / D^{2}\right)^{\alpha}$ seems an appropriate choice. The requirement of finite discomfort and time integrals implies that $3 / 5<\alpha$ $<1$. If again we require finite and non-zero initial value of the jerk at $x= \pm D$, this implies $\alpha=2 / 3$, clearly an admissible value of $\alpha$, although this value is uncomfortably close to the value $\alpha=3 / 5$ at which the discomfort integral diverges. As such, a small "error" in $\alpha$ may potentially lead to a large error in the value of the discomfort integral and a concomitant large error in the Lagrange multiplier $\lambda$. Nevertheless, using the trial function $v=v_{m}\left(1-x^{2} / D^{2}\right)^{2 / 3}$, the Lagrangian becomes

$$
\langle L\rangle=\frac{32 v_{m}^{5}}{9 D^{3}}\left(I_{0}-\frac{10}{3} I_{2}+\frac{25}{9} I_{4}\right)+\frac{2 \lambda D I_{0}}{v_{m}},
$$



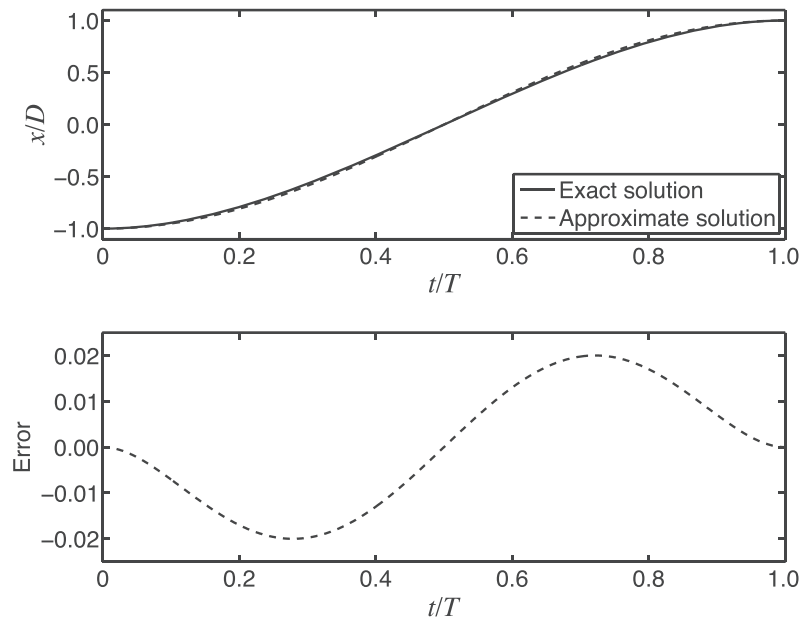

(a)
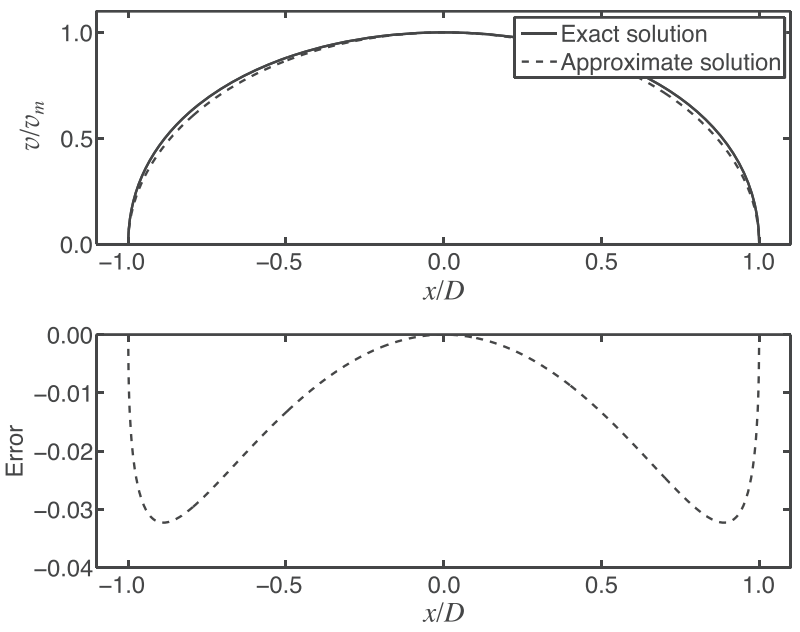

(b)

Fig. 1. Comparison between the approximate and exact solutions. Left graph (distance): Eqs. (30) and (13). Right graph (velocity): $v(x) / v_{m}=\sqrt{1-x^{2} / D^{2}}$ and Eq. (11). The lower figures show the variations of the errors over the interval.

where

$$
I_{n} \equiv \int_{0}^{1} x^{n}\left(1-x^{2}\right)^{-2 / 3} d x=\frac{\Gamma\left(\frac{1}{3}\right) \Gamma\left(\frac{n+1}{2}\right)}{2 \Gamma\left(\frac{n+1}{2}+\frac{1}{3}\right)},
$$

and $\Gamma(x)$ denotes the Gamma function. Variation with respect to $v_{m}$ gives

$$
\Lambda \equiv \frac{\lambda D^{4}}{v_{m}^{6}}=\frac{80}{9}\left(1-\frac{10 I_{2}}{3 I_{0}}+\frac{25 I_{4}}{9 I_{0}}\right)
$$

and using the property that $\Gamma(x+1)=x \Gamma(x)$, this result can be simplified to $\Lambda=320 / 99 \approx 3.23$.

Turning now to a moment analysis of Eq. (19) based on the trial function $v(x)=V_{m}\left(1-x^{2} / D^{2}\right)^{4 / 3}$, the Rayleigh-Ritz procedure prescribes the weight function as $w(x)=\partial V / \partial V_{m}$ $=\left(1-x^{2} / D^{2}\right)^{4 / 3}$, which gives the same result as found above and the same as that obtained using the Galerkin approach. However, in a general moment analysis, we are free to choose the weight function as we please and another possible choice that leads to very simple calculations is $w(x) \propto v(x) \sqrt{v(x)}$ or $w(x)=\left(1-x^{2} / D^{2}\right)^{2}$. In fact, this choice reduces all integrands in the weighted moment to polynomials, thus avoiding the more complicated integrals that arise in the Rayleigh-Ritz and Galerkin procedures. Straightforward calculations then yield $\Lambda=448 / 81 \approx 5.53$. The maximum value of the velocity is determined directly from the travel time condition and is found to be

$$
v_{m}=2 I_{0} \frac{D}{T}=\frac{5 \sqrt{\pi}}{4} \frac{\Gamma(4 / 3)}{\Gamma(11 / 6)} \approx 4.21 D / T .
$$

It is interesting to note that the use of the weight function $w(x)=\left(1-x^{2} / D^{2}\right)^{2}$ avoids the problem of the singularity of the discomfort functional associated with the limit exponent $\alpha=3 / 5$. In fact, using instead this exponent, or the trial function $v(x)=v_{m}^{2}\left(1-x^{2} / D^{2}\right)^{6 / 5}$, the moment method gives

$$
\Lambda=\frac{36}{25}\left(5 K_{0}-\frac{42}{5} K_{2}+\frac{21}{5} K_{4}\right),
$$

where

$$
K_{n}=\int_{0}^{1} x^{n}\left(1-x^{2}\right)^{-2 / 5} d x=\frac{\Gamma\left(\frac{n+1}{2}\right) \Gamma\left(\frac{3}{5}\right)}{2 \Gamma\left(\frac{n+1}{2}+\frac{3}{5}\right)} .
$$

This implies $\Lambda \approx 5.10$ and $v_{m} \approx 3.68 D / T$.

The question of the accuracy of the found solutions can only be settled by a numerical solution of the variational equation, which can be written in the form more suitable for numerical calculations using the normalized variables $V / V_{m} \rightarrow V$ and $x / D \rightarrow x$. This implies the equation

$$
\frac{d^{2}}{d x^{2}}\left(\sqrt{V} \frac{d^{2} V}{d x^{2}}\right)+\frac{1}{4 \sqrt{V}}\left(\frac{d^{2} V}{d x^{2}}\right)^{2}-\frac{\Lambda}{V \sqrt{V}}=0,
$$

subject to the following five (normalized) boundary conditions on the interval $[0,1]: V(1)=d V(1) / d x=0, V(0)=1$, and $d V(0) / d x=d^{3} V(0) / d x^{3}=0$. A numerical solution gives $\Lambda \approx 5.18$ and $v_{m} \approx 3.52 \mathrm{D} / T$, in good agreement with the approximate result obtained by the moment method using the trial function $v(x)=v_{m}^{2}\left(1-x^{2} / D^{2}\right)^{6 / 5}$ and the weight function $w_{T}=V \sqrt{V}$. This is significantly better than the result of the Raleigh-Ritz and Galerkin methods based on the weight function $w(x)=\left(1-x^{2} / D^{2}\right)^{4 / 3}$. A comparison between the approximate solutions and the numerically obtained solution is shown in Fig. 2 and it shows good agreement over the entire interval.

At this point, it is appropriate to emphasize that the variational Eqs. (9) and (19), involving the Lagrange multiplier $\lambda$, from a purely mathematical point of view, can be considered as eigenvalue problems, the multiplier playing the role of the eigenvalue. However, in a variational problem involving a subsidiary condition, the Lagrange multiplier has no physical significance. On the other hand, eigenvalue problems are commonly met in many physical and technical applications where the actual eigenvalue contains important information about the properties of the solution.

It can be argued in the present analysis, based on trial functions involving only one parameter, that a solution could 

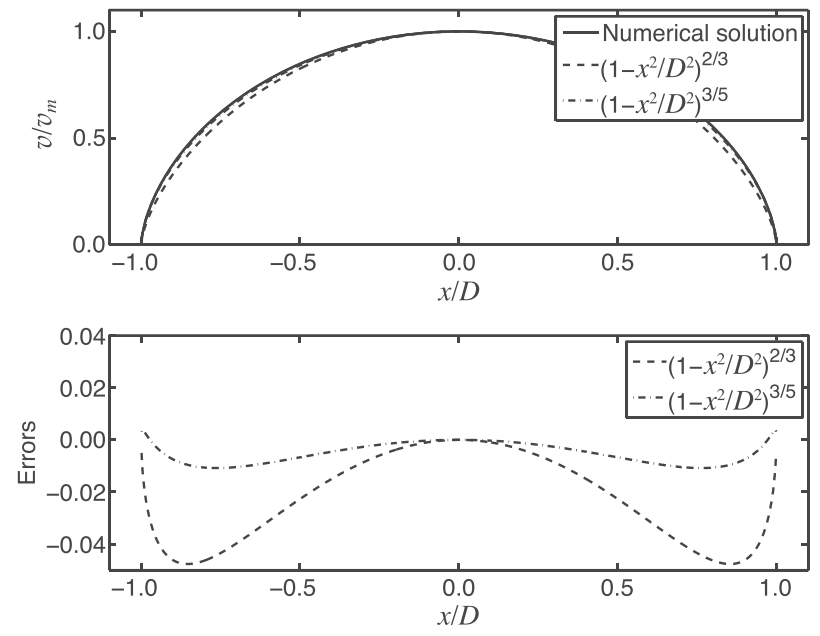

Fig. 2. Comparison between the numerically obtained solution and the trial functions $v_{T}(x) / v_{m}=\left(1-x^{2} / D^{2}\right)^{2 / 3}$ and $v_{T}(x) / v_{m}=\left(1-x^{2} / D^{2}\right)^{3 / 5}$. The lower figure shows the variations of the errors. There is very good agreement; in fact, the best of the trial functions is almost indistinguishable from the numerical solution, except close to the start and end points.

have been determined directly from the subsidiary condition, without even involving a variational formulation and a Lagrange multiplier. However, this "degeneration of the analysis" only occurs in the simplest case of a trial function involving one free parameter and that the form of the trial function be chosen based on properties of the variational functional. Furthermore, an important aspect of our presentation is to illustrate the use of direct variational and moment methods, which may also be used for applications involving eigenvalue problems, where the actual value of the eigenvalue has physical and technical importance. Thus, the determination of the Lagrange multiplier/eigenvalue value in the present problem provides an example of the use of the Rayleigh-Ritz and moment methods for a broad class of other problems. The comparison of the approximate and exact/numerical results for the eigenvalue gives a good indication of the accuracy of the approximate solutions.

Finally, we note that the chosen trial functions imply that the corresponding maximum velocity is larger than that in the first discomfort problem. The reason for this difference becomes obvious when considering the variation of the acceleration in two cases: $a(x)=-v_{m}^{2} x / D^{2}$ and $a(x)=$ $-\left(4 v_{m}^{2} x / 3 D^{2}\right)\left(1-x^{2} / D^{2}\right)^{1 / 3} \quad$ [using the trial function $\left.v_{T}(x)=v_{m}\left(1-x^{2} / D^{2}\right)^{2 / 3}\right]$. Clearly, the journey in the second problem starts out and ends more smoothly with vanishing acceleration/deceleration in order to avoid an infinite jerk at the start and endpoints. This feature has to be compensated for by a higher maximum velocity in order to reach the same travel time as in the first case, and consequently results in a more peaked velocity profile.

\section{CONCLUSION}

The present work provides a short introduction to direct variational methods and the further development to moment methods. The approaches are illustrated by an application to a "discomfort" problem, namely, the problem of minimizing the discomfort experienced during a journey between two points with the travel time held fixed. The approximate solutions illustrate the usefulness of the variational and moment methods and provide simple, yet accurate, approximations. Both methods have the inherent weakness (common to most approximation methods) that it is not possible, a priori, to estimate the accuracy of the obtained approximate solutions. The accuracy of the solutions can only be found by comparison with the exact solutions or with numerically obtained solutions in cases where the investigated equation does not allow an exact analytical solution. It is also demonstrated that a variational result may not necessarily provide the most accurate solution and the reason for this is discussed. The shortcoming of the variational approach in the second problem is due to the fact that the value of the concomitant discomfort functional is mainly determined by the velocity variation at "start and landing," and a small error here strongly influences the total value.

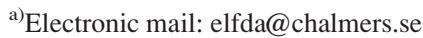

${ }^{1}$ R. P. Feynman, R. B. Leighton, and M. Sands, "The principle of least action" in The Feyman Lectures on Physics (Addison-Wesley, Reading, MA, 1964).

${ }^{2}$ R. Courant and D. Hilbert, Methods of Mathematical Physics (Interscience Publisher, New York, 1953).

${ }^{3}$ I. M. Gelfand and S. V. Fomin, Calculus of Variations (Prentice Hall, Englewood Cliffs, NJ, 1963).

${ }^{4}$ F. Y. M. Wan, Introduction to the Calculus of Variations and Its Applications (Chapman \& Hall, New York, 1995).

${ }^{5}$ L. Komzsik, Applied Calculus of Variations for Engineers (CRC Press/ Taylor and Francis Group, Boca Raton, FL, 2009).

${ }^{6} \mathrm{~B}$. A. Finlayson, The Method of Weighted Residuals and Variational Principles (Academic Press, New York, 1972).

${ }^{7}$ S. H. Schot, "Jerk: The time rate of change of acceleration," Am. J. Phys. 46, 1090-1094 (1978).

${ }^{8}$ D. Anderson, F. Anderson, P. Anderson, A. Billander, M. Desaix, and M. Lisak, "The optimal journey from A to B," Am. J. Phys. 76, 863-866 (2008).

${ }^{9}$ D. J. Kaup and T. K. Vogel, "Quantitative measurement of variational approximations," Phys. Lett. A 362, 289-297 (2007). 